\title{
Influência da lubrificação na maquinabilidade do compósito Tungsténio/Cobre sinterizado (WCu25)
}

\author{
Influence of lubrication on machinability of sintering \\ Tungsten/Copper composite (WCu25) \\ M. Faustino ${ }^{1} \quad$ J. Paulo Davim ${ }^{1}$ \\ Recibido 22 de noviembre de 2011, aceptado 5 de marzo de 2012 \\ Received: November 22, $2011 \quad$ Accepted: March 5, 2012
}

\begin{abstract}
RESUMO
O compósito Tungsténio/Cobre sinterizado (WCu25) é um material utilizado em electrodos para electrorerosão (EDM). O presente trabalho tem como objectivo estudo da influência da lubrificação na maquinabilidade do compósito Tungsténio/Cobre sinterizado (WCu25) utilizando ferramentas de diamante policristalino (PCD). O presente artigo mostra que a utilização lubrificação é fundamental para melhorar a maquinabilidade do material considerando a força e potência de maquinagem, o acabamento de superfície obtido na peça e o desgaste da ferramenta.
\end{abstract}

Palavras chave: maquinabilidade, lubrificação, PCD, acabamento de superficie, desgaste de ferramenta.

\begin{abstract}
The sintering Tungsten/Copper composite (WCu25) is a material used in electrodes for electric discharge machining (EDM). The current work has as objective study the influence of the lubrication in machinability of sintering Tungsten/Copper composite (WCu25) using polycrystalline diamond tools $(P C D)$. The present article shows that the lubrication use is fundamental to improve the machinability of the material considering the machining power and force, the workpiece surface finish and tool wear.
\end{abstract}

Keywords: machinability, lubrication, PCD, surface finish, tool wear.

\section{INTRODUÇÃO}

A definição de maquinabilidade de um material é a maior ou menor facilidade com que o material se pode maquinar por arranque de apara e pode definir-se em função de diversos critérios [1-2]:

- Força e potência de corte;

- Desgaste da ferramenta;

- Acabamento de superfície.

Uma boa maquinabilidade traduz-se num bom acabamento de superfície, em baixa força e potência de corte e também baixo desgaste da ferramenta de corte. Em relação ao controlo das aparas geradas pelo processo de corte, as aparas longas se não se partirem podem interferir com as operações de corte enredando-se na zona de corte obrigando a quebra-aparas.

Portanto, a maquinabilidade não pode ser entendida como uma propriedade do material em sentido estrito porque depende de um conjunto complexo de factores alguns dos quais são externos ao próprio material em estudo [3]

Em respeito às características do material, é preciso ter em conta a sua composição química, microestrutura e propriedades físicas e mecânicas. O tipo de operação e as condições particulares de

1 Departamento de Engenharia Mecânica. Universidade de Aveiro. Campus Santiago, 3810-193. Aveiro, Portugal. E-mail: pdavim@ua.pt 
maquinagem também afectam de modo significativo a maquinabilidade, em particular, o material e a geometria da ferramenta de corte, os parâmetros funcionais do processo de corte (velocidade de corte, avanço e penetramento), a rigidez do sistema, o tipo de corte se continuo ou interrompido, etc.

A maquinagem por electroerosão (EDM) de moldes e matrizes com geometria complexa exige o fabrico de eléctrodos, normalmente em cobre ou grafite, através de tecnologias convencionais como a fresagem e o torneamento. A maquinagem destas ferramentas requer normalmente o fabrico de vários eléctrodos para as diferentes operações de desbaste e acabamento o que aumenta o tempo de fabrico das ferramentas e o respectivo custo [4].

Os materiais normalmente usados em eléctrodos de EDM são de vários tipos nomeadamente cobre, tungsténio, grafite e latão. O cobre é um material extremamente versátil com uma excelente condutividade térmica e eléctrica e devido a estas propriedades é muito requisitado em termos comerciais. Os eléctrodos de cobre-tungsténio $(\mathrm{Cu} / \mathrm{W})$ têm sido normalmente usados na maquinagem de peças de aço e de carboneto de tungsténio devido à alta condutibilidade térmica do cobre, melhor resistência à erosão devido á faísca, baixo coeficiente de expansão térmica e temperatura de fusão alta do tungsténio.

Os eléctrodos de $\mathrm{Cu} / \mathrm{W}$ são mais caros que os tradicionais eléctrodos de Cobre ou de Grafite. No entanto os eléctrodos de $\mathrm{Cu} / \mathrm{W}$ começam a revelar-se como uma mais valia no mercado mundial não só pelo facto de fornecerem um acabamento final à peça melhor como também a durabilidade do próprio eléctrodo é maior que os tradicionais eléctrodos de grafite.

A rugosidade superficial é um parâmetro que tem uma grande influência no comportamento e na funcionalidade dos componentes mecânicos e também nos custos de produção [5-7], constituindo uma importante variável de controlo de qualidade.

O presente trabalho têm como objectivo estudo da influência da lubrificação na maquinabilidade do compósito Tungsténio/Cobre sinterizado (WCu25) utilizando ferramentas de diamante polcristalino (PCD).

\section{METODOLOGIA EXPERIMENTAL}

O compósito de tungsténio/cobre sinterizado (WCu25) ensaiado apresenta baixa dilatação térmica, uma boa condutividade térmica e eléctrica e também uma boa resistência à abrasão.

Os testes de torneamento foram efectuados num torno CNC Kingsbury 50, com uma potência de $18 \mathrm{KW}$ e com uma rotação máxima de $4500 \mathrm{rpm}$. O torno CNC possui um controlador FANUC, uma bucha de aperto hidráulico capaz de fixar peças até 200mm de diâmetro, um armazém de 12 ferramentas e um contraponto de $70 \mathrm{~mm}$ de diâmetro activado também por accionamento hidráulico.

Na execução dos ensaios foram utilizadas pastilhas de corte de PCD (Sandvik TPUN 110304FP-CD10). O porta ferramentas utilizado foi do tipo ISO CTGPL 2020K11 (Sandvik). Na Figura 1 apresentam-se as pastilhas de corte utilizadas na experimentação.

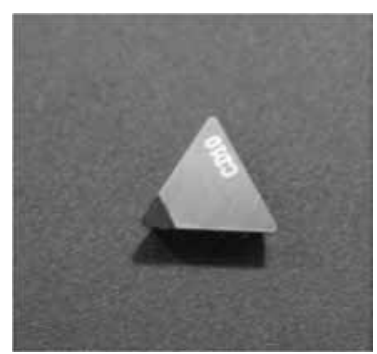

Figura 1. Pastilhas de corte de PCD utilizadas nos ensaios experimentais.

Os parâmetros de corte utilizados nestes testes foram os seguintes: velocidade de corte de $100 \mathrm{~m} / \mathrm{min}$., um avanço de $0,1 \mathrm{~mm} /$ rot e um penetramento de 1 $\mathrm{mm}$. Nos ensaios lubrificados foi utilizado fluído de corte (BP MICRO TREND 231 L, óleo de corte solúvel a $15 \%)$.

As medições das forças de corte, avanço e penetramento, envolvidas no processo de torneamento foram feitas através do dinamómetro piezoeléctrico Kistler® (modelo 9121), -Figura 2- ligado ao amplificador e este por sua vez ao computador, utilizando o software de aquisição de dados Dynoware, adquirindo assim os dados obtidos em formato *. xls para posterior tratamento. 


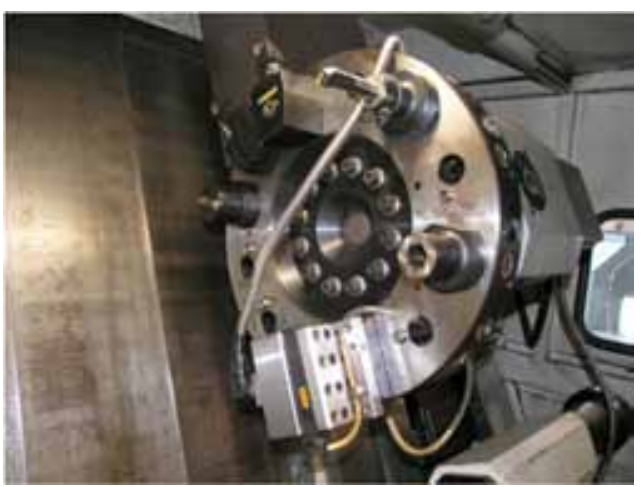

Figura 2. Montagem do dinamómetro no torno CNC.

Os parâmetros de rugosidade utilizados foram a rugosidade média aritmética $(\mathrm{Ra})$ e a amplitude máxima do perfil da rugosidade (Rt) que respeitam a norma ISO/DIS 4287/1E. A medição dos parâmetros de rugosidade (Ra e Rt) foi feita com o equipamento Hommelwerke ${ }^{\circledR}$ T1000 com um comprimento de base (cut off (Lo)) de 0,8 mm e com um comprimento de palpagem (Lt) de 4,8 $\mathrm{mm}$ de acordo com a norma ISO/DIS/ 4287/1E.

O desgaste na face de saída da ferramenta é a medida de desgaste mais usada, uma vez que é mais fácil efectuar a sua medição quantificado pelo parâmetro VB segundo a norma ISO 3685/1993. A medição do desgaste nas pastilhas de corte foi efectuada na sua face de saída, para tal foi usado um microscópio óptico de oficina Mitutoyo ${ }^{\circledR}$ TM-500 com ampliação 30X.

\section{RESULTADOS OBTIDOS E SUA DISCUSSÃO}

Foram efectuados testes de maquinagem no compósito Tungsténio/ cobre sinterizado (WCu25) em estudo segundo a Norma ISO 3685, usando ferramentas com insertos de PCD. Utilizou-se uma velocidade de corte de $100 \mathrm{~m} / \mathrm{min}$., um avanço de $0,1 \mathrm{~mm} /$ rot e um penetramento de $1 \mathrm{~mm}$ em todos os ensaios. Utilizou-se um tempo de corte de aproximadamente de 6 minutos com o objectivo de analisar a influência da lubrificação no desempenho da ferramenta.

\section{Força e potência de maquinagem}

Os valores da força de maquinagem $\left(\mathrm{F}_{\mathrm{m}}\right)$, da potência de corte $(\mathrm{Pc})$ e da força específica de corte
(Ks), podem ser calculados recorrendo às seguintes equações [1-3]:

$$
F_{m}=\sqrt{F_{c}^{2}+F_{a}^{2}+F_{p}^{2}}
$$

sendo $F_{c}$ a força de corte, $F_{a}$ a força de avanço e $F_{p}$ a força de penetramento.

$$
P_{c}=F_{c} \times V_{c}
$$

onde $\mathrm{F}_{\mathrm{c}}$ é a força de corte $\mathrm{e} \mathrm{V}_{\mathrm{c}}$ a velocidade de corte.

$$
K_{s}=\frac{F_{c}}{S}=\frac{F_{c}}{a \times p}
$$

onde $\mathrm{F}_{\mathrm{c}}$ é a força de corte, $\mathrm{S}$ a secção da apara, a o avanço e p o penetramento.

A Figura 3 apresenta a evolução da força de maquinagem $\left(\mathrm{F}_{\mathrm{m}}\right)$ em função do tempo de corte (t) na maquinagem da liga de $\mathrm{WCu} 25$, a seco e com lubrificação.

Observa-se na Figura 3, que a força de maquinagem $\left(F_{m}\right)$ aumenta com o tempo de corte $(t)$. Observa-se também na Figura 3 que o uso de lubrificação no processo de corte diminui consideravelmente a força de maquinagem $\left(\mathrm{F}_{\mathrm{m}}\right)$ em relação ao corte a seco.

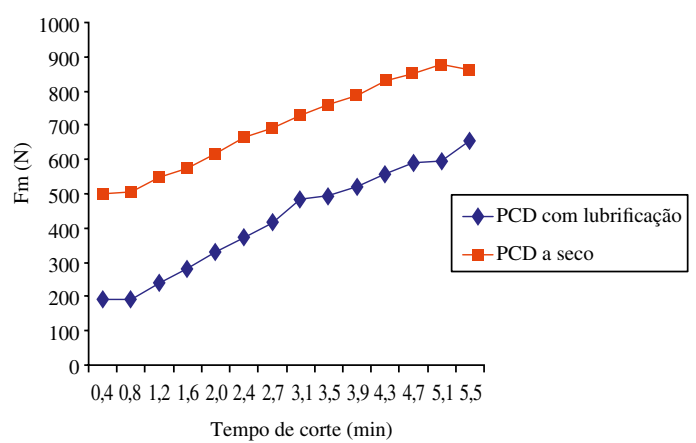

Figura 3. Evolução da força de maquinagem $\left(\mathrm{F}_{\mathrm{m}}\right)$ em função do tempo de corte $(\mathrm{t})$.

A Figura 4. apresenta a evolução da força específica $\left(\mathrm{K}_{\mathrm{s}}\right)$ em função do tempo de corte ( $\mathrm{t}$ ) na maquinagem da liga de $\mathrm{WCu} 25$, a seco e com lubrificação.

Pela observação da Figura 4, a força específica de corte $\left(\mathrm{K}_{\mathrm{s}}\right)$ aumenta ligeiramente com o tempo de corte ( $\mathrm{t}$ ). Observa-se que com o uso de lubrificação 


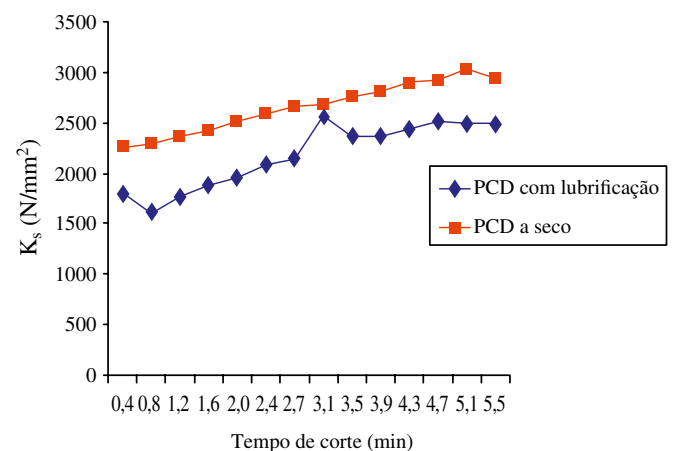

Figura 4. Evolução da força específica $\left(\mathrm{K}_{\mathrm{s}}\right)$ em função do tempo de corte (t).

obtêm-se valores de força especifica de corte $\left(\mathrm{K}_{\mathrm{s}}\right)$ mais baixos em comparação aos testes efectuados a seco.

A Figura 5 apresenta a evolução da potência de corte $\left(\mathrm{P}_{\mathrm{c}}\right)$ em função do tempo de corte $(\mathrm{t})$ na maquinagem da liga de WCu25, a seco e com lubrificação.

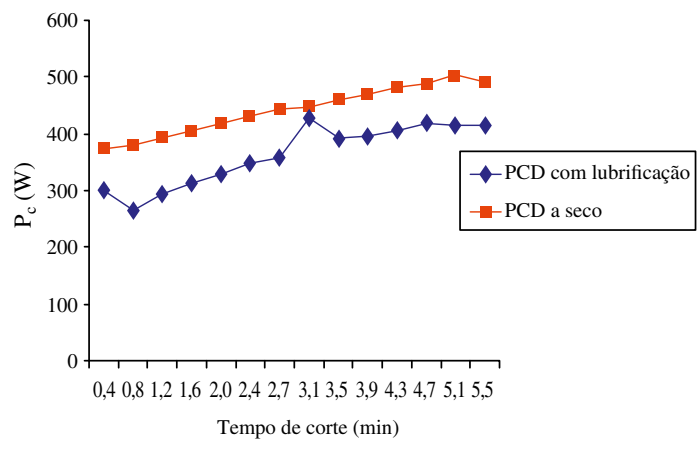

Figura 5. Evolução da potência de corte $\left(\mathrm{P}_{\mathrm{c}}\right)$ em função do tempo de corte (t).

Pela observação da Figura 5, a potência de corte $\left(\mathrm{P}_{\mathrm{c}}\right)$ aumenta com o aumento do tempo de corte $(\mathrm{t})$. Observa-se que com o uso de lubrificação obtêmse valores de potência de corte $\left(\mathrm{P}_{\mathrm{c}}\right)$ mais baixos.

\section{Acabamento de superfície}

A Figura 6 apresenta a evolução da rugosidade média aritmética $(\mathrm{Ra})$ em função do tempo de corte (t) na maquinagem da liga de WCu25, a seco e com lubrificação.

Pela observação da Figura 6, obtém-se valores de rugosidade média aritmética $(\mathrm{Ra})$ mais baixos com o uso de lubrificação em comparação aos ensaios a seco.

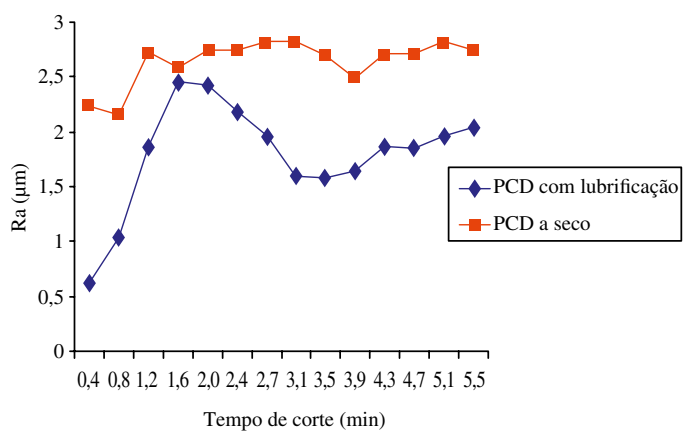

Figura 6. Evolução da rugosidade média aritmética (Ra) em função do tempo de corte (t).

A Figura 7 apresenta a evolução da rugosidade máxima (Rt) em função do tempo de corte (t) na maquinagem da liga de $\mathrm{WCu} 25$, a seco e com lubrificação.

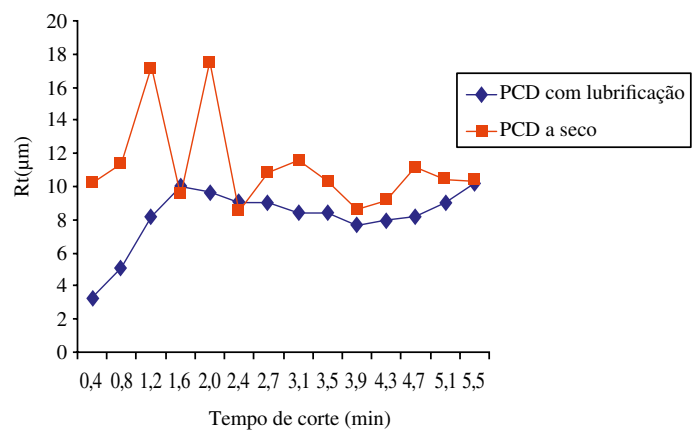

Figura 7. Evolução da rugosidade máxima (Rt) em função do tempo de corte $(\mathrm{t})$.

Pela observação da Figura 7, obtém-se de uma maneira geral, valores de rugosidade máxima (Rt) mais baixos com o uso de lubrificação em comparação aos ensaios a seco.

\section{Desgaste da ferramenta}

A Figura 8 apresenta a evolução do desgaste (VB) em função do tempo de corte (t) na maquinagem da liga de WCu25, a seco e com lubrificação.

Pela observação da Figura 8, o desgaste (VB) aumenta com o aumento do tempo de corte. Observa-se que com o uso de lubrificação obtêm-se valores mais baixos de desgaste (VB).

Na maquinagem da liga $\mathrm{WCu} 25$ com a ferramenta PCD, o uso da lubrificação melhorou fortemente a maquinabilidade no que respeita à força e potência 


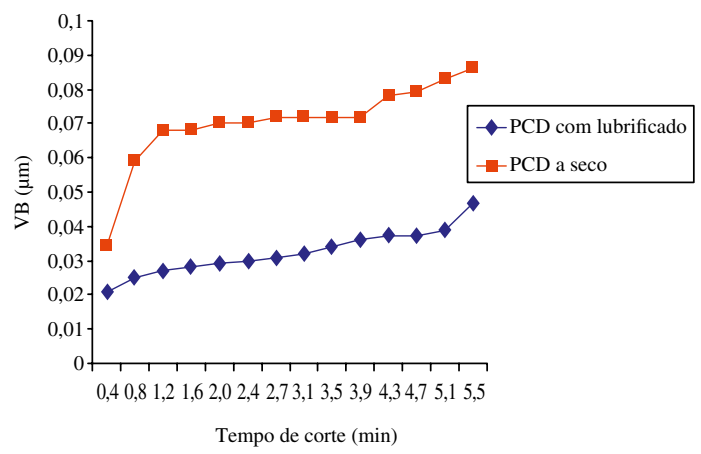

Figura 8. Evolução do desgaste (VB) em função do tempo de corte $(\mathrm{t})$.

de maquinagem, ao acabamento de superfície da peça e ao desgaste da ferramenta.

A Figura 9 mostra o método de medição do desgaste (VB) na face de saída da ferramenta neste tipo de ferramentas.

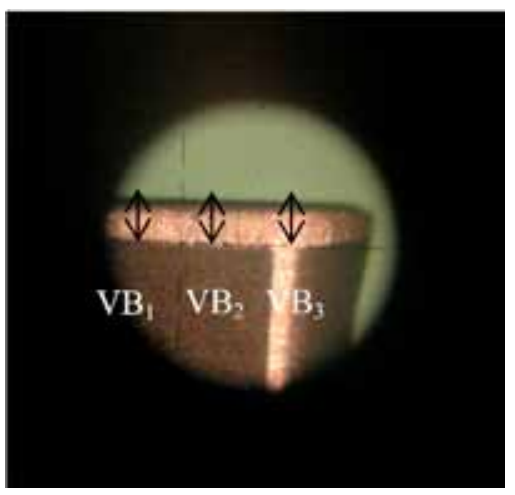

Figura 9. Desgaste VB na face de saída da ferramenta observado no microscópico (30X) segundo a norma ISO 3685/1993, $V B=\frac{V B_{1}+V B_{2}+V B_{3}}{3}$.

\section{CONCLUSÕES}

Os resultados obtidos nesta investigação mostram que a utilização de lubrificação é benéfica para melhorar a maquinabilidade do compósito Tungsténio/Cobre sinterizado (WCu25) com ferramentas de insertos de PCD. Com efeito a temperatura desenvolvida durante a maquinagem deste material é elevada e consequentente o desgaste da ferramenta aumenta significativamente podendo levar a avarias na ferramenta. A lubrificação é importante, também, para melhorar o acabamento de superficie e para reduzir a força e potência de maquinagem.

\section{REFERENCIAS}

[1] J.P. Davim. "Princípios da Maquinagem". Publindústria. Portugal. 2008.

[2] J.P. Davim (Ed.). "Metal Cutting: Research Advances". Nova Publishers. USA. 2010.

[3] M.P. Groover. "Fundamentals of Modern Manufacturing Materials, Process and Systems". Prentice Hall International Editions. USA. 1996.

[4] L. Esperto, M. Santos e A. Osório. "Desempenho de eléctrodos para EDM em cobre/bronze obtidos pelo processo DMLS". Revista Mecânica Experimental, No 11. 2006.

[5] O.B. Abouelatta and J. Mádl. "Surface roughness prediction based on cutting parameters and tool vibrations in turning operations". Journal of Materials Processing Technology. Vol. 118, pp. 269-277. 2001.

[6] G. Petropoulos, J.P. Davim, F. Mata and C. Pandazaras. "New considerations of evaluating the anisotropy of machined surfaces". Journal of Balkan Tribological Association. Vol. 12, Issue 1, pp. 1-6. 2006.

[7] J.P. Davim (Ed.). "Machining: Fundamentals and Recent Advances”. Springer. UK. 2010. 